\title{
The Effect of Pump Coherence on Frequency Conversion and Parametric Amplification
}

\author{
BRUNO CROSIGNANI, PAOLO DI PORTO, URI GANIEL, SALVATORE SOLIMENO, AND AMNON YARIV
}

\begin{abstract}
The consequences of the deviation from ideal coherence of the driving (pump) field in nonlinear processes are examined. A detailed treatment of the processes of parametric amplification and frequency upconversion is presented. The treatment utilizes two different approximation methods to suit the two different physical situations considered. For slow phase variations of the pump field an adiabatic approach is useful, whereas in the treatment of fast phase variations (the nonadiabatic case) an equation describing the time evolution of the $P$ representation is found to be a good starting point. In this latter case, a closed hierarchy of equations for the moments of the photon number distribution in the signal (or idler) mode is found. In particular, we find that the efficiencies of both the amplification and the upconversion processes are reduced as a result of pump incoherence, the reduction becoming more severe in the highly incoherent case. For the frequency converter, the very nature of the process is modified as compared to the ideal case, and this modification is again of particular significance for the highly incoherent pump. Second-order moments are explicitly calculated and some of the statistical properties of the output signals are examined.
\end{abstract}

\section{Introduction}

A $\mathrm{N}$ IMPORTANT class of nonlinear interactions involves the exchange of energy between three nearly harmonic fields of frequencies $\omega_{1}, \omega_{2}$, and $\omega_{0}$ where $\omega_{0}=\omega_{1}+\omega_{2}$. This class of phenomena, usually referred to as parametric interactions, has come to play an important role in a number of areas. As an example we may mention in the microwave field: varactor diode amplifiers and frequency mixers, and in the quantum electronics field: second-harmonic generation, parametric oseillation, and frequency upconversion [1]. Nonlinear parametric interactions have also been found to play an important role in plasma-heating experiments and as loss mechanisms for propagating sound waves.

Turning our attention in particular to the optical regime, two of the most important processes are parametric amplification (PA) and frequency conversion (FC), in

Manuscript received August 13, 1971; revised May 1, 1972. This work was supported by the Advanced Research Projects Agency of the Department of Defense and was monitored by the U. S. Army Research Office (Durham).

B. Crosignani and P. Di Porto were with the California Institute of Technology, Pasadena, Calif. 91109. They are now with the Fondazione Ugo Bordoni, Istituto Superiore P. T., Rome, Italy.

U. Ganiel was with the California Institute of Technology, Pasadena, Calif. 91109. He is now with the Weizmann Institute of Science, Rehovot, Israel.

S. Solimeno was with the California Institute of Technology, Pasadena, Calif. 91109. He is now with the Istituto Elettrotecnico, University of Naples, Naples, Italy.

A. Yariv is with the California Institute of Technology, Pasadena, Calif. 91109. which energy exchange between three electromagnetic modes takes place. The first quantum mechanical treatment of these processes is due to Louisell, Yariv, and Siegman (LYS) [2], who treated the case of a perfectly coherent, monochromatic pump (ideal laser field). In their work, a simple approach is also outlined for obtaining the statistical properties of the amplified, as well as the upconverted signal. The case of the PA was later extensively investigated by Gordon et al. [3], who were able to find the moment-generating function of the output signal and by Mollow and Glauber [4], who gave the complete statistical description in terms of the so-called $P$ representation. More recently, von Foerster and Glauber [5] have considered a traveling-wave PA in which the signal is represented by a more realistic wave packet. In all of these treatments, the pump was assumed to be an external perfectly coherent field and no attention was paid to the fact that an actual laser field always has a finite coherence time.

In what follows, we consider some of the effects of this limited coherence on the efficiency and on the statistical properties of both the PA and the FC processes. Our treatment, as well as those mentioned previously, is based on the approximation that no reaction is exerted on the pump by the two interacting modes. We assume the interaction process to be driven by an external stochastic centered field of constant amplitude as that associated with a real laser operating well above threshold. We explicitly treat the two limiting situations in which the coherence time of the pump is large or small compared with the characteristic times of the PA and $\mathrm{FC}$ processes, when driven by a perfectly coherent field. The lack of coherence of the pump is shown to induce significant changes with respect to the ideal situation. Specifically, the growth rate for the PA output and the efficiency of the FC process are always reduced when the pump is not fully coherent. This degradation becomes extreme in the case of a strongly incoherent pump. In this case even the qualitative nature of the interaction is changed from that of the ideal case. The most striking departure from ideal behavior concerns the gradual deterioration and eventual disappearance of the periodic energy exchange between the low- and high-frequency fields in the FC.

It should be noted that we concentrate in the present investigation on the particular situation in which the pump field drives two specific resonant modes (of frequencies $\omega_{1}$ and $\left.\omega_{2}\right)$. Other situations, such as a singly 
resonant parametric oscillator [6], in which only one of the excited fields is a resonant standing wave, are not included in the present treatment and will be dealt with in a separate investigation.

\section{Formulation of the Problem}

As outlined in the Introduction, we adopt a Hamiltonian that represents a model of two interacting modes of the electromagnetic field [2]. We shall arbitrarily designate one of the modes by subscript 1 and refer to it as the signal and the other will be designated by subscript 2 (the idler). With $a_{r}, a_{r}+(r=1,2)$ being the photon annihilation and creation operators and $\omega_{r}$ their respective frequencies, we write the Hamiltonian for the PA as

$$
\begin{aligned}
H_{\text {amp }}=H_{0}-\hbar[k(t) \exp [- & \left.-i \omega_{0} t\right] a_{1}{ }^{+} a_{2}{ }^{+} \\
& \left.+k^{*}(t) \exp \left[i \omega_{0} t\right] a_{1} a_{2}\right],
\end{aligned}
$$

where $\omega_{0}=\omega_{1}+\omega_{2}$ and for the $\mathrm{FC}$

$$
\begin{aligned}
H_{\text {eonv }}=H_{0}+\hbar[k(t) \exp [ & \left.-i \omega_{0} t\right] a_{1}{ }^{+} a_{2} \\
& \left.+k^{*}(t) \exp \left[i \omega_{0} t\right] a_{1} a_{2}{ }^{+}\right],
\end{aligned}
$$

where $\omega_{0}=\omega_{1}-\omega_{2}$. Here $H_{0}$ is the usual free Hamiltonian

$$
H_{0}=\hbar\left(\omega_{1} a_{1}{ }^{+} a_{1}+\omega_{2} a_{2}{ }^{+} a_{2}\right),
$$

$k(t) \exp \left[-i \omega_{0} t\right]$ representing the coupling between the two modes, which is induced by the pump field.

The equations of motion for the operators are obtained from (1) and (2) utilizing the general relation governing the time evolution of any operator $A$ in the Heisenberg representation $i \hbar(d A / d t)=[A, H]$.

For the PA we have

$$
\begin{aligned}
& d a_{2}{ }^{+} / d t=i \omega_{2} a_{2}{ }^{+}-i k^{*}(t) \exp \left[i \omega_{0} t\right] a_{1}, \\
& d a_{1}{ }^{+} / d t=i \omega_{1} a_{1}{ }^{+}-i k^{*}(t) \exp \left[i \omega_{0} t\right] a_{2}
\end{aligned}
$$

with the corresponding equations for $a_{1}$ and $a_{2}$ obtained as the Hermitian adjoint expressions.

By means of the substitutions

$$
a_{r}=b_{r} \exp \left[-i \omega_{r} t\right], \quad r=1,2
$$

(4a) and (4b) become

$$
\begin{aligned}
& d b_{2}{ }^{+} / d t=-i k^{*}(t) b_{1}, \\
& d b_{1}{ }^{+} / d t=-i k^{*}(t) b_{2} .
\end{aligned}
$$

From (6) and the corresponding Hermitian adjoints, we obtain

$$
\frac{d^{2} b_{r}}{d t^{2}}-\frac{1}{k(t)} \frac{d k(t)}{d t} \frac{d b_{r}}{d t}-|k(t)|^{2} b_{r}=0, \quad(r=1,2) .
$$

These equations can be cast in a different form by means of the substitution

$$
c_{r}=\frac{1}{\sqrt{k(t)}} b_{r}, \quad r=1,2,
$$

thus giving

$$
\ddot{c}_{r}+\left(-|k|^{2}-\frac{3^{2}}{4} \frac{\dot{k}}{k^{2}}+\frac{1}{2} \frac{\dot{k}}{k}\right) c_{r}=0, \quad r=1,2,
$$

where the dot will hereafter indicate a time derivative.

From this point on we shall assume the coupling coeffcient $k(t)$ to be of the form

$$
k(t)=k_{0} \exp [i \phi(t)]
$$

where $\phi(t)$ is a real time-dependent phase. This assumption is certainly a practical one in all cases where the pump field in the parametric process is a laser field, which, well above threshold, has negligible amplitude fluctuation. Equation (8) now becomes

$$
\ddot{c}_{r}+\left(-k_{0}^{2}+\frac{1}{4} \dot{\phi}^{2}+\frac{i}{2} \ddot{\phi}\right) c_{r}=0, \quad r=1,2 .
$$

The FC is treated along analogous lines. Starting from the Hamiltonian (2), performing the substitution as in (5) we obtain

$$
\begin{aligned}
& d b_{1} / d t=-i k(t) b_{2}, \\
& d b_{2} / d t=-i k^{*}(t) b_{1},
\end{aligned}
$$

which can be rewritten as

$$
\begin{gathered}
\ddot{b}_{1}-\frac{\dot{k}}{k} \dot{b}_{1}+|k|^{2} b_{1}=0, \\
\ddot{b}_{2}-\frac{\dot{k}^{*}}{k^{*}} \dot{b}_{2}+|k|^{2} b_{2}=0 .
\end{gathered}
$$

Substituting

$$
c_{1}=\frac{1}{\sqrt{k}} b_{1}, \quad c_{2}=\frac{1}{\sqrt{k^{*}}} b_{2}
$$

and assuming again a constant amplitude coupling, i.e., $k(t)=k_{0} \exp [i \phi(t)]$, we obtain

$$
\begin{aligned}
& \ddot{c}_{1}+\left(k_{0}{ }^{2}+\frac{1}{4} \dot{\phi}^{2}+\frac{i}{2} \ddot{\phi}\right) c_{1}=0, \\
& \ddot{c}_{2}+\left(k_{0}{ }^{2}+\frac{1}{4} \dot{\phi}^{2}-\frac{i}{2} \ddot{\phi}\right) c_{2}=0 .
\end{aligned}
$$

Inspection of (10) and (13) clearly shows that the problem as presented here is not analytically solvable in general. However, for slow (in a sense that will be defined more precisely later) phase variations, an approximation scheme can be adopted that enables one to treat the problem analytically. This treatment will turn out to be relevant for many practical applications of lasers as the pump sources in nonlinear processes.

The other extreme, namely the case where the phase variations are fast, cannot be treated analytically using (10) and (13) as the starting point, and thus has to be approached by completely different methods [7]. This will be done in some detail in Section V.

\section{Solutions for Slow Phase Variations}

In this section we shall utilize the Wentzel-KramersBrillouin (WKB) method [8] to arrive at an approxi- 
mate solution for (10) and (13). An equation of the form

$$
\ddot{x}+\Omega^{2}(t) x=0
$$

in which the adiabatic condition $\left|\Omega / \Omega^{2}\right| \ll 1$ is satisfied, has a pair of approximate independent solutions of the form

$$
x(t) \cong \frac{1}{\sqrt{\Omega(t)}} \exp \left[ \pm i \int_{0}^{t} \Omega\left(t^{\prime}\right) d t^{\prime}\right] .
$$

Equations (10) and (13) are of the form (14), and the adiabatic condition simply corresponds to demanding that the rate of the phase variations of the pump is small compared with $k_{0}$, i.e., $\left|\dot{\phi} / k_{0}\right| \ll 1$.

\section{A. Solution for the PA}

For the PA we have from (10)

$$
\Omega(t)=\sqrt{-k_{0}{ }^{2}+\frac{1}{4} \dot{\phi}^{2}+\frac{i}{2} \dddot{\phi},}
$$

where a definite branch of the square root function must be selected throughout the complex plane (e.g., the principal value). We then have

$c_{r}(t) \cong A_{r} \exp [i \theta(t)]+B_{r} \exp [-i \theta(t)], \quad r=1,2,(17)$

where

$$
\theta(t) \equiv \int_{0}^{t} \Omega\left(t^{\prime}\right) d t^{\prime}
$$

and $A_{r}$ and $B$, are determined from the initial conditions, which are all easily expressed in terms of $a_{r}(t=0) \equiv$ $a_{r_{0}}, a_{r}{ }^{+}(t=0) \equiv a_{r_{0}}{ }^{+}(r=1,2)$, and $\phi(0)$. This leads directly to

$$
\begin{array}{r}
a_{1}(t)=\exp \left[i \frac{\phi(t)}{2}\right] \exp \left[-i \omega_{1} t\right]\left[a_{10} \exp \left[-i \frac{\phi(0)}{2}\right]\right. \\
\left.\cdot \cos \theta(t)+a_{20}{ }^{+} \exp \left[i \frac{\phi(0)}{2}\right] \sin \theta(t)\right] \\
a_{2}(t)=\exp \left[i \frac{\phi(t)}{2}\right] \exp \left[-i \omega_{2} t\right]\left[a_{20} \exp \left[-i \frac{\phi(0)}{2}\right]\right. \\
\left.\cdot \cos \theta(t)+a_{10}{ }^{+} \exp \left[i \frac{\phi(0)}{2}\right] \sin \theta(t)\right] .
\end{array}
$$

From (18) the quantum mechanical expectation values of the photon number operators can be found to be

$$
\begin{aligned}
\left\langle n_{1}(t)\right\rangle & \equiv\left\langle a_{1}{ }^{+}(t) a_{1}(t)\right\rangle=\left\langle n_{10}, n_{20}\left|a_{1}{ }^{+}(t) a_{1}(t)\right| n_{10}, n_{20}\right\rangle \\
& =n_{10} \cos \theta \cos \theta^{*}+\left(1+n_{20}\right) \sin \theta \sin \theta^{*}, \\
\left\langle n_{2}(t)\right\rangle & \equiv\left\langle a_{2}{ }^{+}(t) a_{2}(t)\right\rangle \\
& =n_{20} \cos \theta \cos \theta^{*}+\left(1+n_{10}\right) \sin \theta \sin \theta^{*},
\end{aligned}
$$

where $\left|n_{10}, n_{20}\right\rangle$ is the eigenstate of the photon number operator. Note that if $\phi$ is constant (ideally coherent pump), (19a) and (19b) reduce exactly to the results of LYS [2].

At this point we shall introduce some practical approximations that will enable us to gain better physical in- sight into the nature of the solutions. From (16) we have

$$
\begin{aligned}
\theta(t) & =\int_{0}^{t} \Omega\left(t^{\prime}\right) d t^{\prime} \\
& \simeq i \int_{0}^{t}\left[k_{0}-\frac{1}{8} \frac{\dot{\phi}^{2}\left(t^{\prime}\right)}{k_{0}}-\frac{i}{4 k_{0}} \ddot{\phi}\left(t^{\prime}\right)\right] d t^{\prime} \\
& =i\left[k_{0} t-\frac{1}{8 k_{0}} \int_{0}^{t} \dot{\phi}^{2}\left(t^{\prime}\right) d t^{\prime}\right] \\
& +\frac{1}{4 k_{0}}[\phi(t)-\dot{\phi}(0)],
\end{aligned}
$$

where the fact that $\dot{\phi}^{2}, \ddot{\phi} \ll k_{0}{ }^{2}$ has been used. Using this expression for $\theta(t)$ in (18) we check the boson commutation relations

$$
\begin{array}{r}
{\left[a_{r}(t), a_{r}{ }^{+}(t)\right]=\cos \left(\theta+\theta^{*}\right) \simeq \cos \left[\frac{\phi(t)-\phi(0)}{2 k_{0}}\right]} \\
=1+0\left(\frac{\phi^{2}}{k_{0}{ }^{2}}\right)
\end{array}
$$

to be correct to the lowest significant order. Using (20) and well-known trigonometrical identities, the expressions for the photon numbers (19) can be written as

$$
\begin{aligned}
&\left\langle n_{1}(t)\right\rangle=\frac{1}{2} n_{10}\left\{\cos \left[\frac{1}{2 k_{0}}(\phi(t)-\dot{\phi}(0))\right]\right. \\
&+\left.\cosh \left[2 k_{0} t-\frac{1}{4 k_{0}} \int_{0}^{t} \dot{\phi}^{2}\left(t^{\prime}\right) d t^{\prime}\right]\right\} \\
&+\frac{1}{2}\left(1+n_{20}\right)\left\{-\cos \left[\frac{1}{2 k_{0}}(\phi(t)-\dot{\phi}(0))\right]\right. \\
&\left.+\cosh \left[2 k_{0} t-\frac{1}{4 k_{0}} \int_{0}^{t} \dot{\phi}^{2}\left(t^{\prime}\right) d t^{\prime}\right]\right\},
\end{aligned}
$$

with a similar expression for $\left\langle n_{2}(t)\right\rangle$ (with $n_{10} \leftrightarrow n_{20}$ ).

\section{B. Solution for the FC}

For the FC we have from (13)

$$
\begin{aligned}
& \Omega_{1}(t)=\sqrt{k_{0}{ }^{2}+\frac{1}{4} \phi^{2}+\frac{i}{2} \ddot{\phi}}, \\
& \Omega_{2}(t)=\sqrt{k_{0}{ }^{2}+\frac{1}{4} \phi^{2}-\frac{i}{2} \ddot{\phi}}
\end{aligned}
$$

and

$c_{r} \cong A_{r} \exp \left[i \theta_{r}(t)\right]+B_{r} \exp \left[-i \theta_{r}(t)\right], \quad r=1,2$

where

$$
\theta_{r}(t) \equiv \int_{0}^{t} \Omega_{r}\left(t^{\prime}\right) d t^{\prime} .
$$

Proceeding as before we obtain

$$
\begin{aligned}
a_{1}(t)= & \exp \left[i \frac{\phi(t)}{2}\right] \exp \left[-i \omega_{1} t\right]\left[a_{10} \exp \left[-i \frac{\phi(0)}{2}\right]\right. \\
& \left.\cdot \cos \theta_{1}(t)-i a_{20} \exp \left[i \frac{\phi(0)}{2}\right] \sin \theta_{1}(t)\right],
\end{aligned}
$$




$$
\begin{gathered}
a_{2}(t)=\exp \left[-i \frac{\phi(t)}{2}\right] \exp \left[-i \omega_{2} t\right]\left[a_{20} \exp \left[i \frac{\phi(0)}{2}\right]\right. \\
\left.\cdot \cos \theta_{2}(t)-i a_{10} \exp \left[-i \frac{\phi(0)}{2}\right] \sin \theta_{2}(t)\right]
\end{gathered}
$$

and

$$
\begin{aligned}
& \left\langle n_{1}(t)\right\rangle=n_{10} \cos \theta_{1} \cos \theta_{1}{ }^{*}+n_{20} \sin \theta_{1} \sin \theta_{1}{ }^{*} \\
& \left\langle n_{2}(t)\right\rangle=n_{20} \cos \theta_{2} \cos \theta_{2}{ }^{*}+n_{10} \sin \theta_{2} \sin \theta_{2}{ }^{*},
\end{aligned}
$$

which can again be seen to reduce to the LYS [2] result if $\phi$ is constant. The $\theta_{r}$ can again be approximated by

$$
\theta_{1,2}(t) \cong k_{0} t+\frac{1}{8 k_{0}} \int_{0}^{t} \dot{\phi}^{2}\left(t^{\prime}\right) d t^{\prime} \pm \frac{i}{4 k_{0}}[\dot{\phi}(t)-\dot{\phi}(0)]
$$

and hence

$$
\begin{aligned}
& \left\langle n_{1}(t)\right\rangle=\frac{1}{2} n_{10}\left\{\cos \left[2 k_{0} t+\frac{1}{4 k_{0}} \int_{0}^{t} \dot{\phi}^{2}\left(t^{\prime}\right) d t^{\prime}\right]\right. \\
& \left.+\cosh \left[\frac{1}{2 k_{0}}(\phi(t)-\dot{\phi}(0))\right]\right\}+\frac{1}{2} n_{20}\left\{-\cos \left[2 k_{0} t\right.\right. \\
& \left.\left.+\frac{1}{4 k_{0}} \int_{0}^{t} \dot{\phi}^{2}\left(t^{\prime}\right) d t^{\prime}\right]+\cosh \left[\frac{1}{2 k_{0}}(\phi(t)-\dot{\phi}(0))\right]\right\}
\end{aligned}
$$

and a similar expression for $\left\langle n_{2}(t)\right\rangle$ (with $n_{10} \leftrightarrow n_{20}$ ). As before, the commutation relations satisfy

$$
\left[a_{r}(t), a_{r}^{+}(t)\right] \cong \cosh \left[\frac{\dot{\phi}(t)-\dot{\phi}(0)}{2 k_{0}}\right]=1+0\left(\frac{\dot{\phi}^{2}}{k_{0}^{2}}\right),
$$

which again are correct to the lowest significant order.

\section{Statistical Averages}

The solutions obtained in the previous section represent a deterministic approach in the sense that the coupling coefficient $k(t)$ has been treated as a prescribed function of time. In practice, $k(t)$ is a random function, hence all the final results must be expressed in terms of appropriate statistical averages. With this in mind, we quote here a result of statistical physics known as the method of semiinvariants [9]. Considering a random variable $x$, the function $\langle\exp (i u x)\rangle_{\mathrm{av}}$ can be described by

$$
\langle\exp (i u x)\rangle_{\mathrm{av}}=\exp \left[\sum_{n=1}^{\infty} \frac{(i u)^{n}}{n !} M_{n}\right],
$$

where the quantities $M_{n}$ are expressed in terms of the moments $\left\langle x^{s}\right\rangle_{\mathrm{av}}(s \leq n)$ and \langle\rangle$_{\mathrm{av}}$ stands for an ensemble average [9]. In particular, when $x$ is a Gaussian variable, (31) reduces to

$$
\langle\exp (i u x)\rangle_{\mathrm{av}}=\exp \left[-\frac{1}{2} u^{2}\left\langle x^{2}\right\rangle_{\mathrm{av}}\right] .
$$

The quantities of interest to us are $\left\langle\left\langle n_{1}(t)\right\rangle\right\rangle_{\mathrm{av}}$ and $\left\langle\left\langle n_{2}(t)\right\rangle\right\rangle_{\mathrm{av}}$. We observe that the random variable of interest, $(22)$ and $(29)$, is $\dot{\phi}(t)$, which represents the instantaneous deviation of the pump frequency from the central value of $\omega_{0}$. It is a realistic assumption to regard $\dot{\phi}(t) \equiv \omega(t)$ as a Gaussian variable [10]. This enables us to evaluate immediately the averages of all terms containing expres- sions of the form $\exp [u \omega(t)]$. On the other hand, $\omega^{2}(t)$ is not a Gaussian variable and evaluation of the averages involving it would require, in principle, a knowledge of all the moments of its statistical distribution (31). In the framework of the present calculation, however, it has been assumed all along that $\dot{\phi} \equiv \omega \ll k_{0}$, so we shall keep in (31) only terms up to second order, i.e.,

$$
\langle\exp (i u x)\rangle_{\mathrm{av}} \cong \exp \left[i u M_{1}-\frac{u^{2}}{2} M_{2}\right]
$$

where

$$
M_{1} \equiv\langle x\rangle_{\mathrm{av}}, \quad M_{2} \equiv\left\langle x^{2}\right\rangle_{\mathrm{av}}-\langle x\rangle_{\mathrm{av}}{ }^{2} .
$$

\section{A. Average Photon Numbers}

Utilizing (32) and (33), we obtain from (22) for the PA

$$
\begin{aligned}
& \left\langle\left\langle n_{1}(t)\right\rangle\right\rangle_{\mathrm{av}} \\
& =\frac{1}{2} n_{10}\left\{\exp \left[\frac{1}{16 k_{0}{ }^{2}} \int_{0}^{t} \int_{0}^{t} G^{2}\left(t^{\prime}-t^{\prime \prime}\right) d t^{\prime} d t^{\prime \prime}\right]\right. \\
& \left.\cdot \cosh \left[\left(2 k_{0}-\frac{G(0)}{4 k_{0}}\right) t\right]+\exp \left[-\frac{1}{4 k_{0}{ }^{2}}(G(0)-G(t))\right]\right\} \\
& +\frac{1}{2}\left(1+n_{20}\right)\left\{\exp \left[\frac{1}{16 k_{0}{ }^{2}} \int_{0}^{t} \int_{0}^{t} G^{2}\left(t^{\prime}-t^{\prime \prime}\right) d t^{\prime} d t^{\prime \prime}\right]\right. \\
& \left.\cdot \cosh \left[\left(2 k_{0}-\frac{G(0)}{4 k_{0}}\right) t\right]-\exp \left[-\frac{1}{4 k_{0}{ }^{2}}(G(0)-G(t))\right]\right\}(34)
\end{aligned}
$$

and a corresponding equation for $\left\langle\left\langle n_{2}(t)\right\rangle\right\rangle_{a v}$ (with $1 \leftrightarrow 2$ ). In deriving (34), $\omega(t)$ has been assumed to be a stationary Gaussian variable and thus the following factorization property has been utilized

$$
\left\langle\omega^{2}\left(t^{\prime}\right) \omega^{2}\left(t^{\prime \prime}\right)\right\rangle_{\mathrm{av}}=2\left\langle\omega\left(t^{\prime}\right) \omega\left(t^{\prime \prime}\right)\right\rangle_{\mathrm{uv}}{ }^{2}+\left\langle\omega^{2}(0)\right\rangle_{\mathrm{av}}{ }^{2},
$$

and

$$
G\left(t^{\prime}-t^{\prime \prime}\right) \equiv\left\langle\omega\left(t^{\prime}\right) \omega\left(t^{\prime \prime}\right)\right\rangle_{\mathrm{av}} .
$$

The quantity $G$ can be expressed in terms of the power spectrum of the pump. This is done in the Appendix.

A similar procedure leads to the following result for the FC

$$
\begin{aligned}
& \left\langle\left\langle n_{1}(t)\right\rangle\right\rangle_{\mathrm{av}}=\frac{1}{2} n_{10}\left\{\exp \left[\frac{1}{4 k_{0}{ }^{2}}(G(0)-G(t))\right]\right. \\
& +\exp \left[-\frac{1}{16 k_{0}{ }^{2}} \int_{0}^{t} \int_{0}^{t} G^{2}\left(t^{\prime}-t^{\prime \prime}\right) d t^{\prime} d t^{\prime \prime}\right] \\
& \left.\cdot \cos \left[\left(2 k_{0}+\frac{G(0)}{4 k_{0}}\right) t\right]\right\} \\
& +\frac{1}{2} n_{20}\left\{\exp \left[\frac{1}{4 k_{0}{ }^{2}}(G(0)-G(t))\right]\right. \\
& -\exp \left[-\frac{1}{16 k_{0}{ }^{2}} \int_{0}^{t} \int_{0}^{t} G^{2}\left(t^{\prime}-t^{\prime \prime}\right) d t^{\prime} d t^{\prime \prime}\right] \\
& \left.\cdot \cos \left[\left(2 k_{0}+\frac{G(0)}{4 k_{0}}\right) t\right]\right\},
\end{aligned}
$$


and a corresponding equation for $\left\langle\left\langle n_{2}(t)\right\rangle\right\rangle_{\mathrm{av}}$ (with $1 \leftrightarrow 2$ ). In both (34) and (37) the terms of the form exp $[ \pm$ $\left.\left(1 / 4 k_{0}{ }^{2}\right)(G(0)-G(t))\right]$ are instantaneous, i.e., their effect is not cumulative in time, so that to the lowest significant order $\exp \left[ \pm\left(1 / 4 k_{0}{ }^{2}\right)(G(0)-G(t))\right] \cong 1$. We note here that the Manley-Rowe relations [11] are satisfied by our solutions, since we obtain ${ }^{1}$

$$
\begin{aligned}
\left\langle\left\langle n_{1}(t)\right\rangle\right\rangle_{\mathrm{av}} & \mp\left\langle\left\langle n_{2}(t)\right\rangle\right\rangle_{\mathrm{av}} \\
& =\left(n_{10} \mp n_{20}\right) \exp \left\{\mp \frac{1}{4 k_{0}{ }^{2}}[G(0)-G(t)]\right\}
\end{aligned}
$$

where the lower and upper signs refer to the PA and the FC, respectively.

The effect of the phase variations in the case of the PA is clearly brought out by inspection of (34); it is clear that the growth rate of the average photon number (which in the absence of phase fluctuations would simply be $2 k_{0}$ ) is reduced by a (positive) quantity $G(0) / 4 k_{0}$ that is proportional to the mean square value of the frequency fluctuation $\omega(t)$. In the case of the $\mathrm{FC}$, the partial incoherence of the pump has two interesting effects. First, the rate of energy exchange between the two modes increases, but the efficiency of the exchange process is reduced. Second, comparing (37) with the coherent pump case $[2$, eqs. $(70),(71)]$ where complete energy exchange between the signal and the idler fields occurs periodically, we note that in the present case the efficiency of the exchange decreases with time (owing to the multiplying factor

$$
\left.\exp \left[-\frac{1}{16 k_{0}{ }^{2}} \int_{0}^{t} \int_{0}^{t} G^{2}\left(t^{\prime}-t^{\prime \prime}\right) d t^{\prime} d t^{\prime \prime}\right]\right)
$$

until asymptotically the exchange becomes insignificant and the photon numbers in both modes become practically equal

$$
\left\langle\left\langle n_{1}(t)\right\rangle\right\rangle_{\mathrm{av}} \cong\left\langle\left\langle n_{2}(t)\right\rangle\right\rangle_{\mathrm{av}} \cong \frac{1}{2}\left(n_{10}+n_{20}\right) .
$$

It is interesting to note that this equipartition effect takes place regardless of how small the pump incoherence is. One must bear in mind, however, that for this to happen in practice the time needed may be longer than the actual interaction time, in which case this effect will not be observed. However, we would like to stress that all the conclusions arrived at in this section have more than a mere theoretical significance, since although derived from some smallness assumptions with regard to the phase variations, they point towards clear trends of the physical processes involved. In Section $\mathrm{V}$ this trend will be brought out in a more pronounced manner by considering the case of high incoherence of the pump field.

\footnotetext{
${ }^{1}$ The presence of the term

$$
\exp \left[\frac{1}{16 k_{0}^{2}} \int_{0}^{t} \int_{0}^{t} G^{2}\left(t^{\prime}-t^{\prime \prime}\right) d t^{\prime} d t^{\prime \prime}\right]
$$
}

can be checked not to affect this conclusion practically.

\section{B. Further Consideration of Statistical Properties}

In order to gain better insight into the statistical properties of the system, one needs to calculate the higher moments of the photon number operator. A point that is important in this context is the choice of the initial state of the physical system. Up to this point, the states $\left|n_{10}, n_{20}\right\rangle$, which are eigenstates of the photon number operator, have been used. A better choice for the calculation of the moments is to use the coherent states [12], which are, in general, the most appropriate description of laser fields, especially well above threshold. The coherent states are defined as the eigenstates of the annihilation operator $a$, with an eigenvalue $\alpha$

$$
a|\alpha\rangle=\alpha|\alpha\rangle \text {. }
$$

In evaluating the first moments $\left(\left\langle\left\langle n_{r}(t)\right\rangle\right\rangle_{\mathrm{av}}\right)$ such an approach leads to the results we have obtained previously. In calculating higher order moments this would no longer be true, hence we shall use the coherent states in the following calculations.

We shall concern ourselves with the calculations for the FC only. ${ }^{2}$ Starting from

$$
\begin{aligned}
\left\langle n_{1}(t)\right\rangle & \equiv\left\langle\alpha_{1}, \alpha_{2}\left|a_{1}{ }^{+}(t) a_{1}(t)\right| \alpha_{1}, \alpha_{2}\right\rangle \\
\left\langle n_{1}{ }^{2}(t)\right\rangle & \equiv\left\langle\alpha_{1}, \alpha_{2}\left|a_{1}{ }^{+}(t) a_{1}(t) a_{1}{ }^{+}(t) a_{1}(t)\right| \alpha_{1}, \alpha_{2}\right\rangle,
\end{aligned}
$$

it follows directly that

$$
\begin{aligned}
\left\langle n_{1}{ }^{2}(t)\right\rangle & =\left\langle\alpha_{1}, \alpha_{2}\left|\left[a_{1}{ }^{+}(t)\right]^{2}\left[a_{1}(t)\right]^{2}\right| \alpha_{1}, \alpha_{2}\right\rangle \\
& +\left\langle\alpha_{1}, \alpha_{2}\left|a_{1}{ }^{+}(t) a_{1}(t)\right| \alpha_{1}, \alpha_{2}\right\rangle \\
& =\left\langle n_{1}(t)\right\rangle^{2}+\left\langle n_{1}(t)\right\rangle .
\end{aligned}
$$

Taking the statistical average, we have

$$
\begin{aligned}
\left\langle\left\langle n_{1}{ }^{2}(t)\right\rangle\right\rangle_{\mathrm{av}}=\left\langle\left\langle n_{1}(t)\right\rangle\right\rangle_{\mathrm{av}} & +\left\langle\left\langle n_{1}(t)\right\rangle^{2}\right\rangle_{\mathrm{av}} \\
=\left\langle\left\langle n_{1}(t)\right\rangle\right\rangle_{\mathrm{av}} & +\left\langle\left\langle n_{1}(t)\right\rangle\right\rangle_{\mathrm{av}}{ }^{2} \\
& +\left(\left\langle\left\langle n_{1}(t)\right\rangle^{2}\right\rangle_{\mathrm{av}}-\left\langle\left\langle n_{1}(t)\right\rangle\right\rangle_{\mathrm{av}}{ }^{2}\right),
\end{aligned}
$$

where the term in circular parenthesis on the right-hand side (RHS) represents the deviation from the case of coherent (Poissonian) light. Assuming mode 1 to be initially empty, which represents the situation in a practical upconverter, i.e.,

$$
a_{10}\left|\alpha_{1}, \alpha_{2}\right\rangle=0
$$

we obtain

$$
\begin{aligned}
\left\langle\left\langle n_{1}(t)\right\rangle^{2}\right\rangle_{\mathrm{av}}-\left\langle\left\langle n_{1}(t)\right\rangle\right\rangle_{\mathrm{av}}{ }^{2}= & \left|\alpha^{2}\right|^{4}\left(\left\langle\sin ^{2} \theta_{1} \sin ^{2} \theta_{1}{ }^{*}\right\rangle_{\mathrm{av}}\right. \\
& \left.-\left\langle\sin \theta_{1} \sin \theta_{1}{ }^{*}\right\rangle_{\mathrm{av}}{ }^{2}\right),
\end{aligned}
$$

where (26a) has been used. Evaluating (46) explicitly

\footnotetext{
${ }^{2}$ For the PA, detailed treatment of the statistical properties for the coherent pump case has been presented by Mollow and Glauber [3]. Their general result was that the signal coherence deteriorates in time even for an ideally coherent pump.
} 
and keeping terms only to the lowest significant order, we have

$$
\begin{aligned}
& \left\langle\sin ^{2} \theta_{1} \sin ^{2} \theta_{1}{ }^{*}\right\rangle_{\mathrm{av}}-\left\langle\sin \theta_{1} \sin \theta_{1}{ }^{*}\right\rangle_{\mathrm{av}}{ }^{2} \\
& =\frac{1}{8}+\frac{1}{8} \exp \left[-\frac{1}{4 k_{0}{ }^{2}} \int_{0}^{t} \int_{0}^{t} G^{2}\left(t^{\prime}-t^{\prime \prime}\right) d t^{\prime} d t^{\prime \prime}\right] \\
& \cdot \cos \left[\left(4 k_{0}+\frac{G(0)}{2 k_{0}}\right) t\right] \\
& -\frac{1}{4} \exp \left[-\frac{1}{8 k_{0}{ }^{2}} \int_{0}^{t} \int_{0}^{t} G^{2}\left(t^{\prime}-t^{\prime \prime}\right) d t^{\prime} d t^{\prime \prime}\right] \\
& \cdot \cos ^{2}\left[\left(2 k_{0}+\frac{G(0)}{4 k_{0}}\right)_{t}\right] .
\end{aligned}
$$

From (47), (46), and (44) and (37) the variance of the number of photons can be calculated directly. We shall write here explicitly the asymptotic result that holds for times in which the equipartition effect (39) takes place. In this case the normalized variance of the upconverted light is

$$
\epsilon^{2} \equiv \frac{\left\langle\left\langle n^{2}(t)\right\rangle\right\rangle_{\mathrm{av}}-\langle\langle n(t)\rangle\rangle_{\mathrm{av}}{ }^{2}}{\langle\langle n(t)\rangle\rangle_{\mathrm{av}}{ }^{2}} \cong \epsilon_{\mathrm{coh}}{ }^{2}+\frac{1}{2},
$$

where $\epsilon^{2}$ coh is the normalized variance of coherent (Poissonian) light. Equation (48) represents an intermediate behavior between Poissonian and thermal (Gaussian) light, for which

$$
\epsilon_{\text {thermal }}^{2}=\epsilon_{\mathrm{coh}}^{2}+1 .
$$

\section{Solutions for Fast Phase Variations}

As stated previously, (10) and (13) cannot be solved analytically in general, once the phase variations $(\dot{\phi}, \ddot{\phi})$ become too large. In this section we present a different approach that enables us to treat the problem under quite general conditions. However, having treated the slow variation case previously, we concentrate on the other limiting case. A short version of this treatment has been recently reported [7].

Our starting point is again the Hamiltonian (1) for the PA or (2) for the FC. We use a recent formulation [13] for writing down the equation of motion of the so-called $P$ representation, using the Hamiltonian of the system. We obtain the following equations in the interaction representation ${ }^{3}$

$$
i(\partial / \partial t) P\left(\left\{\alpha_{k}^{*}, \alpha_{k}\right\} ; t\right)=\Lambda(t) P\left(\left\{\alpha_{k}^{*}, \alpha_{k}\right\} ; t\right),
$$

where for the PA

\footnotetext{
$s$ We should comment here that the equation derived in [13] is written in the Schrödinger picture. It is straightforward to obtain the corresponding equation in the interaction representation. This is done simply by starting from the equation of motion for the density operator $\rho$ in the interaction representation and diagonalizing $\rho$ by means of a corresponding function $P$.
}

$$
\begin{aligned}
\Lambda(t) & =-k(t)\left(\frac{\partial^{2}}{\partial \alpha_{1} \partial \alpha_{2}}-\alpha_{2}{ }^{*} \frac{\partial}{\partial \alpha_{1}}-\alpha_{1}{ }^{*} \frac{\partial}{\partial \alpha_{2}}\right) \\
& -k^{*}(t)\left(-\frac{\partial^{2}}{\partial \alpha_{1}{ }^{*} \partial \alpha_{2}{ }^{*}}+\alpha_{2} \frac{\partial}{\partial \alpha_{1}{ }^{*}}+\alpha_{1} \frac{\partial}{\partial \alpha_{2}{ }^{*}}\right)
\end{aligned}
$$

and for the $\mathrm{FC}$

$$
\begin{aligned}
\Lambda(t)=k^{*}(t)\left(\alpha_{2} * \frac{\partial}{\partial \alpha_{1}{ }^{*}}\right. & \left.-\alpha_{1} \frac{\partial}{\partial \alpha_{2}}\right) \\
& +k(t)\left(\alpha_{1}^{*} \frac{\partial}{\partial \alpha_{2}{ }^{*}}-\alpha_{2} \frac{\partial}{\partial \alpha_{1}}\right) .
\end{aligned}
$$

As before $k(t)$ is the coupling coefficient that represents the coupling between modes 1 and 2 induced by the pump field. The advantage of introducing the $P$-representation formalism lies in the fact that it furnishes a direct way for evaluating the complete statistical properties of the system. In particular, it enables one to express statistical averages of operators of the form

$$
\prod_{k}\left(a_{k}^{*}\right)^{m} a_{k}^{n}
$$

as integrals of the form

$$
\begin{aligned}
& \left\langle\left\langle\prod_{k}\left(a_{k}{ }^{+}\right)^{m} a_{k}{ }^{n}\right\rangle\right\rangle_{\mathrm{av}} \\
& \quad=\int P\left(\left\{\alpha_{k}^{*}, \alpha_{k}\right\} ; t\right) \prod_{k}\left(\alpha_{k}^{*}\right)^{m} \alpha_{k}^{n} d^{2} \alpha_{k} .
\end{aligned}
$$

Equation (53) will turn out to be extremely useful in what follows.

From (50) we obtain

$$
\begin{aligned}
P\left(\left\{\alpha_{k}{ }^{*}, \alpha_{k}\right\} ; t\right)=P\left(\left\{\alpha_{k}{ }^{*}, \alpha_{k}\right\} ; 0\right) & \\
& -i \int_{0}^{t} \Lambda\left(t^{\prime}\right) P\left(\left\{\alpha_{k}^{*}, \alpha_{k}\right\} ; t^{\prime}\right) d t^{\prime}
\end{aligned}
$$

and resubstituting this expression for $P$ into the RHS and taking the time derivative leads to

$$
\begin{aligned}
(\partial / \partial t) P\left(\left\{\alpha_{k}{ }^{*}, \alpha_{k}\right\} ; t\right) & =-i \Lambda(t) P\left(\left\{\alpha_{k}^{*}, \alpha_{k}\right\} ; 0\right) \\
& -\int_{0}^{t} \Lambda(t) \Lambda\left(t^{\prime}\right) P\left(\left\{\alpha_{k}^{*}, \alpha_{k}\right\} ; t^{\prime}\right) d t^{\prime},
\end{aligned}
$$

where $\Lambda(t)$ is given by (51) and (52) for the cases of the PA and FC, respectively. Taking the ensemble average we obtain

$$
\begin{array}{r}
(\partial / \partial t) \bar{P}\left(\left\{\alpha_{k}^{*}, \alpha_{k}\right\} ; t\right)=-i\langle\Lambda(t)\rangle_{\mathrm{av}} P\left(\left\{\alpha_{k}{ }^{*}, \alpha_{k}\right\} ; 0\right) \\
-\int_{0}^{t}\left\langle\Lambda(t) \Lambda\left(t^{\prime}\right) P\left(\left\{\alpha_{k}{ }^{*}, \alpha_{k}\right\} ; t^{\prime}\right)\right\rangle_{\mathrm{av}} d t^{\prime},
\end{array}
$$

where $\bar{P}$ denotes the ensemble average of $P$. At this point we note that the fluctuating part of $\Lambda(t)$ is $k(t)$ [or $k^{*}(t)$ ], which we assume to describe a centered (i.e., $\langle k(t)\rangle_{\mathrm{av}}=0$ ) stationary stochastic process. Hence the first term on the RHS of (55) vanishes. Furthermore, we assume that the coupling between modes 1 and 2 induces only small changes in $P$ during a characteristic time period $t_{c}$, which describes the decay rate of the correlation function 
$\langle\Lambda(t) \Lambda(0)\rangle_{a v}{ }^{4}$ This enables us to simplify the integrand on the RHS of (55) and obtain for $t>t_{c}$

$$
\begin{aligned}
(\partial / \partial t) & \bar{P}\left(\left\{\alpha_{k}{ }^{*}, \alpha_{k}\right\} ; t\right) . \\
& =-\int_{0}^{t}\langle\Lambda(t) \Lambda(t-s)\rangle_{\mathrm{av}} \bar{P}\left(\left\{\alpha_{k}{ }^{*}, \alpha_{k}\right\} ; t-s\right) d s .
\end{aligned}
$$

It is important to note that this Markov-process assumption was not made in the adiabatic case, treated in Section III, hence the results obtained below cannot be applied there.

We now consider the moments

$\langle m, n ; t\rangle$

$$
\equiv \int d^{2} \alpha_{1} d^{2} \alpha_{2}\left(\alpha_{1}{ }^{*} \alpha_{1}\right)^{m}\left(\alpha_{2}{ }^{*} \alpha_{2}\right)^{n} \bar{P}\left(\left\{\alpha_{k}{ }^{*}, \alpha_{k}\right\} ; t\right),
$$

which satisfy the equation

$$
\begin{aligned}
& (d / d t)\langle m, n ; t\rangle \\
& =-\int_{0}^{t} d s \int d^{2} \alpha_{1} d^{2} \alpha_{2}\left(\alpha_{1}{ }^{*} \alpha_{1}\right)^{m}\left(\alpha_{2}{ }^{*} \alpha_{2}\right)^{n}\langle\Lambda(t) \Lambda(t-s)\rangle_{\mathrm{av}} \\
& \cdot \bar{P}\left(\left\{\alpha_{k}{ }^{*}, \alpha_{k}\right\} ; t-s\right),
\end{aligned}
$$

where $(56)$ has been used. Utilizing repeated integration by parts leads to the following expression

$$
\begin{aligned}
& (d / d t)\langle m, n ; t\rangle \\
& =-\int_{0}^{t} d s \int d^{2} \alpha_{1} d^{2} \alpha_{2} \tilde{P}\left(\left\{\alpha_{k}{ }^{*}, \alpha_{k}\right\} ; t-s\right) \\
& \cdot\langle\Lambda(t-s) \Lambda(t)\rangle_{\mathrm{av}}{ }^{+}\left(\alpha_{1}{ }^{*} \alpha_{1}\right)^{m}\left(\alpha_{2}{ }^{*} \alpha_{2}\right)^{n} .
\end{aligned}
$$

In (59), $\langle\Lambda(t-s) \Lambda(t)\rangle_{\mathrm{av}}{ }^{\dagger}$ defines an operator that is obtained from $\langle\Lambda(t-s) \Lambda(t)\rangle_{\mathrm{av}}$ by the substitution $\partial / \partial \alpha_{i} \rightarrow-\partial / \partial \alpha_{i}$.

In writing $\langle\Lambda(t-s) \Lambda(t)\rangle_{\mathrm{av}}{ }^{\dagger}$ explicitly, we note that averages of the type $\langle k(t-s) k(t)\rangle_{\mathrm{av}}$ or $\left\langle k^{*}(t-s) k^{*}(t)\right\rangle_{\mathrm{av}}$ vanish, and we define

$$
g(s) \equiv 2\left\langle k(t) k^{*}(t-s)\right\rangle_{a v} .
$$

Using (51), (52), and (60) we obtain from (59)

$$
\begin{aligned}
(d / d t)\langle m, n ; t\rangle= & \int_{0}^{t} d s g(s)\left\{m^{2} n^{2}\langle m-1, n-1 ; t-s\rangle\right. \\
& +m^{2}(2 n+1)\langle m-1, n ; t-s\rangle \\
& +n^{2}(2 m+1)\langle m, n-1 ; t-s\rangle \\
& +m^{2}\langle m-1, n+1 ; t-s\rangle \\
& +n^{2}\langle m+1, n-1 ; t-s\rangle \\
& +(2 m n+m+n)\langle m, n ; t-s\rangle\}
\end{aligned}
$$

for the PA and

\footnotetext{
- This is true in the spirit of the present calculation that assumes fast variations of $k(t)$, or equivalently, $t_{e} \ll|k(t)|^{-1}$. As a reference for the application of the so-called Born approximation to the evolution of the $\bar{P}$ (see [14]).
}

$$
\begin{aligned}
(d / d t)\langle m, n ; t\rangle= & \int_{0}^{t} d s g(s)\left\{m^{2}\langle m-1, n+1 ; t-s\rangle\right. \\
& +n^{2}\langle m+1, n-1 ; t-s\rangle \\
& -(2 m n+m+n)\langle m, n ; t-s\rangle\}
\end{aligned}
$$

for the FC.

Equations (61a) and (61b) constitute the central result of this section. It is easy to realize that they define a elosed hierarchy of integrodifferential equations. so that any moment $\langle m, n ; t\rangle$ can be calculated from them once $g(s)$ is given. Note that our results depend only on $g(s)$, which is essentially the Fourier transform of the power spectrum of the pump field (see the Appendix). This, however, is a direct consequence of the Markovprocess assumption mentioned above.

We note that (61a) and (61b) are of the general form

$$
\begin{aligned}
\frac{d}{d t} y(t) & =\int_{0}^{t} d s g(s) F(t-s)=\int_{0}^{t} d s g(t-s) F(s) \\
& =\int_{0}^{\infty} d s \tilde{g}(t-s) F(s),
\end{aligned}
$$

where we have defined

$$
\tilde{g}(x)= \begin{cases}g(x), & x \geq 0 \\ 0, & x<0 .\end{cases}
$$

Taking the Laplace transform defined as

$$
f(p) \equiv \int_{0}^{\infty} \exp [-p t] f(t) d t,
$$

we get from (62)

$$
p y(p)-y(t=0)=g(p) F(p) .
$$

With this result we can transform (61a) and (61b) immediately into

$$
\begin{aligned}
p\langle m, n ; p\rangle-\langle m, n ; t=0\rangle=g(p)\left\{m^{2} n^{2}\langle m-1, n-1 ; p\rangle\right. \\
\quad+m^{2}(2 n+1)\langle m-1, n ; p\rangle \\
\quad+n^{2}(2 m+1)\langle m, n-1 ; p\rangle \\
\quad+m^{2}\langle m-1, n+1 ; p\rangle+n^{2}\langle m+1, n-1 ; p\rangle \\
\quad+(2 m n+m+n)\langle m, n ; p\rangle\}
\end{aligned}
$$

for the PA and

$$
\begin{aligned}
p\langle m, n ; p\rangle-\langle m, n ; t & =0\rangle \\
=g(p)\left\{m^{2}\langle m-1, n\right. & +1 ; p\rangle+n^{2}\langle m+1, n-1 ; p\rangle \\
& -(2 m n+m+n)\langle m, n ; p\rangle\}
\end{aligned}
$$

for the $\mathrm{FC}$, which define a closed hierarchy of algebraic equations. We shall be interested in the quantities 


$$
\begin{aligned}
\left\langle\left\langle n_{1}(t)\right\rangle\right\rangle_{\mathrm{av}} & =\langle 1,0 ; t\rangle \\
\left\langle\left\langle n_{2}(t)\right\rangle\right\rangle_{\mathrm{av}} & =\langle 0,1 ; t\rangle \\
\left\langle\left\langle n_{1}{ }^{2}(t)\right\rangle\right\rangle_{\mathrm{av}} & =\langle 2,0 ; t\rangle+\langle 1,0 ; t\rangle \\
\left\langle\left\langle n_{2}{ }^{2}(t)\right\rangle\right\rangle_{\mathrm{av}} & =\langle 0,2 ; t\rangle+\langle 0,1 ; t\rangle,
\end{aligned}
$$

which can be evaluated from (66a) and (66b) once $g(s)$ is specified.

In the following, we take the pump field to have a Lorentzian power spectrum [10], so that

$$
g(s)=2 k_{0}^{2} \exp [-\gamma s] .
$$

In this case the moments (67) can be evaluated in closed form. The expressions, although straightforward, are cumbersome and will not be reproduced here. However, as has already been noted, we are dealing in this section with a situation where $k_{0}$ is much smaller than the inverse of the characteristic variation time of the pump $t_{c}$, or equivalently

$$
\eta \equiv k_{0} / \gamma \ll 1 .
$$

Under this condition, rather simple expressions for the moments (67) can be written down applying suitable approximations. Assuming the idler mode to be initially empty $\left(\left\langle n_{20}\right\rangle=0\right)$, we obtain for the PA

$\left\langle\left\langle n_{1}(t)\right\rangle\right\rangle_{\mathrm{av}}=\frac{1}{2}\left(\left\langle n_{10}\right\rangle+1\right) \exp \left(4 \eta k_{0} t\right)+\frac{1}{2}\left\langle n_{10}\right\rangle-\frac{1}{2}$

and

$$
\begin{aligned}
\left\langle\left\langle n_{1}{ }^{2}(t)\right\rangle\right\rangle_{\mathrm{av}} & =\frac{1}{6}\left(2\left\langle n_{10}{ }^{2}\right\rangle-5\left\langle n_{10}\right\rangle+5\right) \\
& +\frac{1}{2}\left(\left\langle n_{10}{ }^{2}\right\rangle-3\right) \exp \left(4 \eta k_{0} t\right) \\
& +\frac{1}{6}\left(\left\langle n_{10}{ }^{2}\right\rangle+5\left\langle n_{10}\right\rangle+2\right) \exp \left(12 \eta k_{0} t\right) .
\end{aligned}
$$

For the FC, assuming $\left\langle n_{10}\right\rangle=0$, the same approximations lead to

$$
\left\langle\left\langle n_{1}(t)\right\rangle\right\rangle_{\mathrm{av}}=\frac{1}{2}\left\langle n_{20}\right\rangle\left[1-\exp \left(-4 \eta k_{0} t\right)\right]
$$

and

$$
\begin{gathered}
\left\langle\left\langle n_{\mathrm{t}}{ }^{2}(t)\right\rangle\right\rangle_{\mathrm{av}}=\frac{1}{6}\left(2\left\langle n_{20}{ }^{2}\right\rangle-\left\langle n_{20}\right\rangle\right)-\frac{1}{2}\left\langle n_{20}{ }^{2}\right\rangle \exp \left(-4 \eta k_{0} t\right) \\
+\frac{1}{6}\left(\left\langle n_{20}{ }^{2}\right\rangle+\left\langle n_{20}\right\rangle\right) \exp \left(-12 \eta k_{0} t\right) .
\end{gathered}
$$

Equation (70) for the PA can be contrasted with (34), which also reproduces the coherent pump case by substituting $G=0$. It is easily seen that in the present case the growth rate is drastically reduced, being multiplied by a factor $\eta \ll 1$.

In the case of the FC, the effect is still more dramatic, since the very nature of the process is changed. Contrasting (37) with (72) we see that in the present case the oscillatory character of the process is completely damped. The efficiency of energy exchange between the two modes is so drastically reduced that the maximum possible conversion efficiency is 50 percent, since asymptotically

$$
\left\langle\left\langle n_{1}(t)\right\rangle\right\rangle_{\mathrm{av}} \cong \frac{1}{2}\left\langle n_{20}\right\rangle
$$

In most practical cases the interaction times involved are such that $\eta k_{0} t \ll 1$, so that this equipartition situation is not reached and the conversion efficiency is therefore much lower.

\section{Conclustons}

The effects of pump coherence on optical parametric amplifiers and frequency converters have been examined in detail. Two cases have been examined in particular, namely those of "slow" and "fast" frequency fluctuations. The first case has been treated using the adiabatic approximation, whereas for the second case it was found advantageous to resort to a different technique, utilizing the equation describing the time evolution of the $P$ representation. Important deviations from the ideally coherent pump situation have been found. In both cases, one finds that the growth rate of the signal field of the PA is reduced, rather slightly for the slowly fluctuating pump and much more significantly for the fast fluctuating pump. For the FC, the rate of energy exchange is modified in both cases as shown qualitatively in Fig. 1. While for the fast fluctuating pump case this is obvious, the periodic nature of the exchange process being completely damped out, in the other case the modification of this process depends on the detailed nature of the correlation function of the frequency fluctuation. The rate of the energy exchange is enhanced somewhat, while the efficiency of the process is reduced.

Finally, we would like to mention that although our detailed treatment concerns only the optical PA and FC, they are two examples of a large family of nonlinear processes to which similar considerations can be applied.

\section{Appendix}

A laser field of constant amplitude and random phase can be written as

$$
E(t)=E_{0} \exp \left[i \omega_{0} t\right] \exp \left[i \int_{0}^{t} \omega\left(t^{\prime}\right) d t^{\prime}\right] .
$$

The Wiener-Kintchine theorem enables one to express the power spectrum $S(\omega)$ in terms of the correlation function of the field $\left\langle E(t) E^{*}(0)\right\rangle_{\mathrm{av}}$ as

$$
\left\langle E(t) E^{*}(0)\right\rangle_{\mathrm{av}}=\int S(\Omega) \exp [i \Omega t] d \Omega .
$$

Using (74) and introducing the normalized spectrum $s(\omega)$ $=S(\omega) /\left|E_{0}\right|^{2}$, one can write

$$
\begin{aligned}
\left\langle\exp \left[i \int_{0}^{t} \omega\left(t^{\prime}\right) d t^{\prime}\right]\right. & \\
& =\int s\left(\Omega+\omega_{0}\right) \exp [i \Omega t] d \Omega .
\end{aligned}
$$

Assuming the distribution function of instantaneous frequency fluctuations $\omega(t)$ to follow the Gaussian station- 


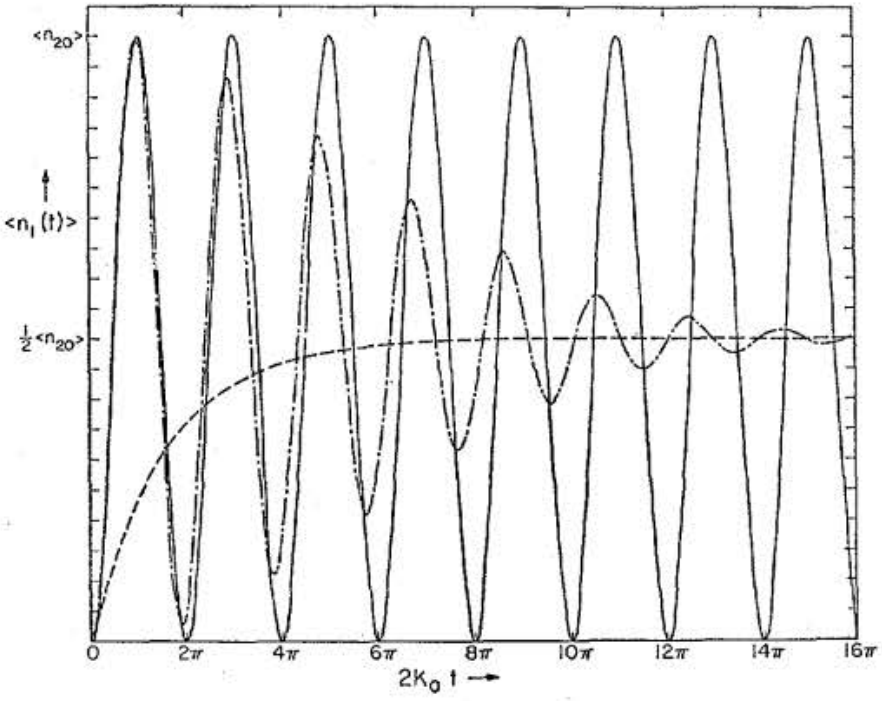

Fig. 1. Typical time behavior of the average number of upconverted photons for the cases. Continuous line-ideally coherent pump-see (37) with $G=0$. Dashed-dotted line-see (37). Dashed line-see noncoherent pump (72).

law, (76) becomes

$$
\begin{aligned}
\exp \left[-\frac{1}{2} \int_{0}^{t} \int_{0}^{t} G\left(t^{\prime}\right.\right. & \left.\left.-t^{\prime \prime}\right) d t^{\prime} d t^{\prime \prime}\right] \\
& =\int s\left(\Omega+\omega_{0}\right) \exp [i \Omega t] d \Omega
\end{aligned}
$$

Equation (77) immediately yields

$$
\begin{aligned}
s\left(\Omega+\omega_{0}\right) & =\frac{1}{2 \pi} \int \exp [-i \Omega t] \\
\cdot \exp & {\left[-\frac{1}{2} \int_{0}^{t} \int_{0}^{t} G\left(t^{\prime}-t^{\prime \prime}\right) d t^{\prime} d t^{\prime \prime}\right] d t . }
\end{aligned}
$$

Furthermore, utilizing the identity

$$
\int_{0}^{t} \int_{0}^{t} G\left(t^{\prime}-t^{\prime \prime}\right) d t^{\prime} d t^{\prime \prime}=2 \int_{0}^{t}(t-\tau) G(\tau) d \tau
$$

ary one easily obtains

$$
G(t)=-\frac{d^{2}}{d t^{2}} \ln \left[\int s\left(\Omega+\omega_{0}\right) \exp [i \Omega t] d \Omega\right] .
$$

\section{ACKNOWLEDGMENT}

The authors wish to thank J. Pearson for some useful discussions.

\section{References}

[1] For an extensive review on parametric oscillators see: S. E. Harris, "Tunable optical parametric oscillators," Proc. IEEE, vol. 57, pp. 2096-2113, Dec. 1969.

[2] W. H. Louisell, A. Yariv, and A. E. Siegman, "Quantum fluctuations and noise in parametric processes, I," Phys. Rev., vol. 124, pp. 1646-1654, Dec. 1961.

[3] J. P. Gordon, W. H. Louisell, and L. R. Walker, "Quantum fluctuation and noise in parametric processes, II," Phys. Rev., vol. 129, pp. 481-485, Jan. 1963.

[4] B. R. Mollow and R. J. Glauber, "Quantum theory of parametric amplification, I and II," Phys. Rev., vol. 160, pp. 1076-1108, Aug. 1967.

[5] T. von Foerster and R. J. Glauber, "Quantum theory of light propagation in amplifying media," Phys. Rev. A, vol. 3, pp. 1484-1511, Apr. 1971.

[6] J. F. Young, R. B. Miles, S. E. Harris, and R. W. Wallace, "Pump linewidth requirement for optical parametric oscillations," J. Appl. Phys., vol. 42, p. 497, 1971.

[7] B. Crosignani, U. Ganiel, S. Solimeno, and A. Yariv, "Some consequences of pump coherence on energy exchange in nonlinear optical processes," Phys. Rev. (Lett.), vol. 27, pp. 237-239, Aug. 1971.

[8] For an extensive review of the WKB method, see for example: J. Heading, An Introduction to Phase Integral Methods. London: Methuen, 1962.

[9] R. Brout and P. Carruthers, Lectures on the Many Electron Problem. New York: Interscience, 1962.

[10] R. J. Glauber, in Quantum Optics and Electronics. Les Houches, France: Univ. Grenoble, 1964; also C. H. DeWitt et al., Eds. New York: Gordon and Breach, 1965.

[11] J. M. Manley and H. E. Rowe, "General energy relations in nonlinear reactances," Proc. IRE (Corresp.), vol. 47, pp. 2115-2116, Dec. 1959.

[12] R. J. Glauber, "Coherent and incoherent states of the radiation field," Phys. Rev., vol. 131, pp. 2766-2788, Sept. 1963. The reader may consult this reference for an extensive treatment of the coherent states and the $P$ representation.

[13] a. B. Crosignani, S. Solimeno, and P. Di Porto, "Master equation for the $P$ representation," Phys. Rev. (Lett.), vol. 26, pp. 1130-1132, May 1971. (Single mode case.) b. B. Crosignani, U. Ganiel, S. Solimeno, and P. Di Porto, "An equation of motion for the $P$ representation," Phys. Rev. A, vol. 4, pp. 1570-1574, Oct. 1971 (general case.)

[14] a. F. Haake, "On a non-Markoffian master equation," $Z$. Phys., vol. 223, pp. 353-375, 1969.

b. B. Crosignani, P. Di Porto, and S. Solimeno, "Quantum theory of a randomly modulated harmonic oscillator," Phys. Rev., vol. 186, pp. 1342-1347, Oct. 1969. 